\title{
Responsabilidad Socio - Ambiental en Proyectos Hidroeléctricos: El Caso Río Zamora - Santiago
}

\author{
P. Martínez \\ L. Ochoa \\ J. L. Espinoza \\ Corporación Eléctrica del Ecuador CELEC E.P., Unidad de Negocio HIDROPAUTE
}

Resumen- CELEC EP-HIDROPAUTE, opera las centrales hidroeléctricas Mazar (170 MW), Molino $(1075 \mathrm{MW})$, supervisa la construcción del proyecto Sopladora (487 MW) y administra la ejecución de los estudios Cardenillo (593 MW) y Río ZamoraSantiago ( $>5500$ MW prefactibilidad).

El Gobierno Nacional, a través del Ministerio de Electricidad y Energía Renovable-MEER, establece como política que los proyectos de generación eléctrica deberán impulsar el desarrollo sostenible de las comunidades vecinas, asegurando la calidad, seguridad, gestión ambiental y responsabilidad social.

Durante los estudios del Proyecto Hidroeléctrico Río Zamora-Santiago (PHRZS), se identificaron 94 comunidades donde se aplicaron encuestas, asambleas, visitas domiciliarias y entrevistas, lo que permitió el contacto con líderes comunitarios y la población en general para informar y recibir sus expectativas.

La mayor expectativa que genera un proyecto de este tipo tiene que ver con la satisfacción de necesidades básicas insatisfechas. Se determinó que el $\mathbf{4 2} \%$ de la población tiene como prioridad el desarrollo de servicios básicos, entre los cuales destaca el servicio de energía eléctrica.

La Gestión Socio-ambiental de HIDROPAUTE, considera la implementación de proyectos de desarrollo territorial, tales como agua potable, alcantarillado y electrificación. Para el caso del PHRZS se ha contemplado la ejecución de proyectos de electrificación rural y alumbrado público, a través de convenios con la empresa distribuidora, CENTROSUR.

Hasta julio de 2013 se incorporan 205 nuevos clientes, 820 beneficiarios. Se tiene previsto una segunda etapa que incluyen 120 nuevos clientes y alrededor de 500 beneficiarios.

Los proyectos de generación eléctrica, si bien pueden cumplir la normativa vigente, nogarantizan un adecuado desarrollo de los territorios donde éstos se asientan, mientras que las comunidades exigen mejoras en su nivel de vida. Ello demanda acciones públicas, con un enfoque intersectorial, que trasciende la responsabilidad de "construir un proyecto" y lleva a los promotores a involucrarse en procesos de desarrollo a través de la prestación de servicios básicos.

Palabras clave- Generación hidroeléctrica, responsabilidad social, servicios básicos.

Abstract- CELEC EP-HIDROPAUTE operates the Mazar Hydroelectric Power Plant (170 MW) and the Molino Hydroelectric Power Plant (1,075 MW), oversees construction of the Sopladora Project (487 MW) and manages studies for the Cardenillo Project (593 MW) and the Rio Zamora Santiago Project $(>5,500 M W$ pre-feasibility).

The National Government, acting through the Ministerio de Electricidad y Energía Renovable - MEER (The Ministry of Electricity and Renewable Energy) has made it a policy that electric power generation projects should stimulate the sustainable development of neighbouring communities, ensuring quality, security, environmental management and social responsibility.

During studies for the Rio Zamora-Santiago Hydroelectric Project (PHRZS), 94 local communities were identified and contacted through surveys, meetings, domestic visits, and interviews, making it possible to contact community leaders and the population in general to inform them about the project and understand their expectations.

The highest expectation created by a project of this nature involves satisfying basic unmet needs. It was determined that $42 \%$ of the population considers the development of basic utilities to be a priority, and electrical power has been emphasized in particular.

Social and Environmental Management of the HIDROPAUTE Business Unit has planned 
the implementation of certain development projects, such as drinking water, sewerage, and electrification. Where PHRZS is concerned, the execution of rural electrification and public lighting has been planned through agreements reached with CENTROSUR, the energy distribution company.

As of July 2013, 205 new clients and 820 beneficiaries have been incorporated. A second stage is expected to follow, which will include 120 new clients and around 500 beneficiaries.

While such electric power generation projects may very well comply with current regulations, they do not guarantee an adequate development of the areas where they are implemented, given the demand of local communities to improve their standard of life. This requires public incentive, with a cross-sector focus, which goes beyond the responsibility of "building a project" and leads its developers to become involved in the development process through the provision of basic utility services.

Index Terms - Hydroelectric power generation, social responsibility, basic utilities.

\section{INTRODUCCIÓN}

Una de las estrategias establecidas por el Estado ecuatoriano para promover el desarrollo del país es el cambio de la Matriz Eléctrica. Para ello, el Ministerio de Electricidad y Energía Renovable - MEER, entre los principales aspectos de su gestión, fomenta el incremento de la participación de energías renovables y la soberanía energética, lo cual se refleja en la construcción de 8 centrales hidroeléctricas, el primer parque eólico del Ecuador continental; ejecución de 5 proyectos de energías renovables para las islas Galápagos y estudios de proyectos de generación hidroeléctrica y geotérmica.

Con ello se proyecta, a partir del 2016, transformar la matriz eléctrica, actualmente con un peso similar de hidroelectricidad y termoelectricidad, a una con un aporte de energías renovables superior al $90 \%$, básicamente hidroelectricidad.

Para una implementación integral de todos estos proyectos, el MEER establece como política que las empresas promotoras de proyectos eléctricos deberán impulsar el desarrollo sostenible de las comunidades de la región en donde se implanten, con normas relacionadas a sus procesos para asegurar la calidad, gestión ambiental, seguridad y que se rijan por criterios de inclusión y responsabilidad social.

Por otro lado el Consejo Nacional de Electricidad CONELEC, elaboró el Plan Nacional de Electrificación con sustento en cuatro ejes, uno de ellos el Eje Inclusivo, tiene como uno de sus objetivos "Armonizar el desarrollo y operación de la infraestructura del sector eléctrico con las expectativas de las comunidades".

La Empresa Pública Estratégica Corporación Eléctrica del Ecuador (CELEC EP) está a cargo de la generación y transmisión de energía eléctrica dentro del país. Una de sus Unidades de Negocio, CELEC EP - HIDROPAUTE, opera las centrales hidroeléctricas Mazar (170MW), Molino (1075MW), supervisa la construcción del proyecto Sopladora (487MW) y administra la ejecución de los estudios Cardenillo (593MW) y Río Zamora Santiago (>5500MW, según prefactibilidad). CELEC EP - HIDROPAUTE paralelamente con el avance de los estudios, la ejecución y operación de los proyectos de generación a su cargo promueve el desarrollo sustentable de los territorios ejecutando planes con la participación de las diferentes autoridades, actores sociales, entidades estatales y públicas, de tal forma que se brinde atención a las comunidades de las áreas de influencia de sus proyectos. En este artículo se describe como CELEC EP contribuye a garantizar el acceso universal al servicio de electrificación en dichas comunidades.

\section{POLITICAS DE GESTIÓN AMBIENTAL Y RESPONSABILIDAD SOCIAL DE CELEC EP-HIDROPAUTE}

CELEC EP- HIDROPAUTE consciente de la responsabilidad de implementar procedimientos para el cuidado del Ambiente y el desarrollo de los territorios del área de influencia de sus proyectos, considera 4 componentes en su cadena de valor que conforman los ejes de la Gestión Ambiental y Responsabilidad Social de la Empresa.

\section{Eje 1: Apoyo al Desarrollo Territorial}

Una de las estrategias fundamentales de la inversión pública está encaminada a satisfacer la dotación de bienes y servicios esenciales, dotación que permite mejorar y salvaguardar las capacidades de la sociedad en su conjunto y de los individuos que la conforman.

En el desarrollo de los proyectos hidroeléctricos a su cargo, CELEC EP-HIDROPAUTE ha establecido acciones encaminadas al fortalecimiento de las comunidades del área de influencia. 
Las líneas de acción establecidas son:

- Fortalecimiento a los procesos productivos comunitarios

- Apoyo a cantones y parroquias del área de influencia de los proyectos hidroeléctricos

- Proyectos de electrificación y alumbrado público

El objetivo de estas acciones es contribuir al desarrollo socioeconómico de las comunidades del área de influencia, a través de:

- Fomentar y fortalecer la organización micro empresarial y la modernización de la producción en el área de influencia

- Apoyar el desarrollo de tecnologías de producción amigables con el ambiente

- Mejorar los sistemas de infraestructura sanitaria de las comunidades

- Mejorar el acceso a los servicios básicos

Para el cumplimiento de lo planteado, HIDROPAUTE ha implementado programas con las comunidades y organizaciones, del área de influencia de sus proyectos hidroeléctricos. La fotografía 1 muestra una visita desarrollada por líderes comunitarios de las comunidades del Proyecto Hidroeléctrico Río Zamora-Santiago para conocer los proyectos productivos desarrollados alrededor del Proyecto Paute-Mazar

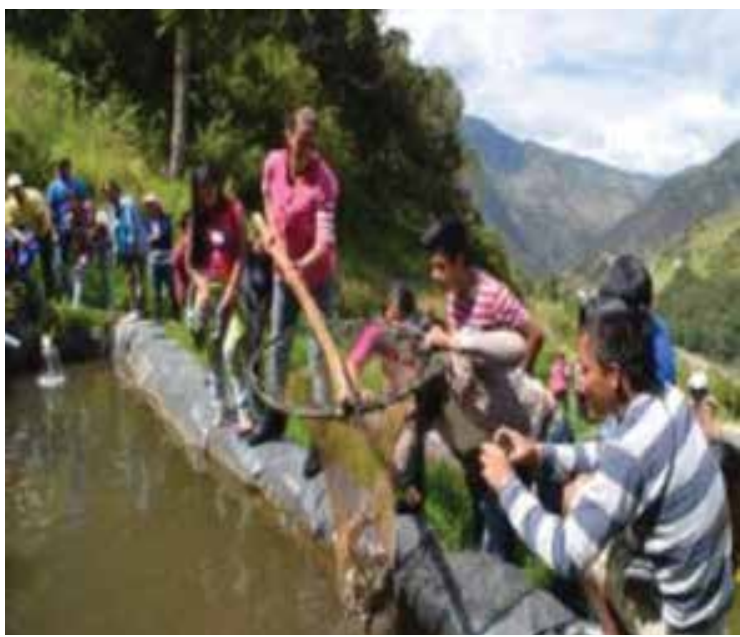

Fotografía 1: Visita al proyecto comunitario de truchas Arcoiris. Proyecto Hidroeléctrico Mazar. Fuente: PHRZS

Para la ejecución de los proyectos de electrificación, que se detallan más adelante, se han firmado convenios de cooperación interinstitucional con la empresa eléctrica distribuidora de energía Centro Sur, encargada de brindar el servicio en las áreas donde se desarrollan los proyectos hidroeléctricos.
Eje 2: Educomunicación para el Desarrollo Sostenible

Este eje busca fortalecer y diversificar las capacidades y potencialidades individuales y sociales, y promover una ciudadanía participativa y crítica. En coordinación con el Ministerio de Educación, CELEC EP-HIDROPAUTE realiza actividades encaminadas a la capacitación y constante información de los habitantes de su área de influencia en biodiversidad, gestión de desechos, cuidado de recursos naturales entre otros. La interacción con la población a través de la educomunicación permite complementar y afianzar los proyectos de desarrollo socio ambiental y orientar sobre el manejo de los proyectos hidroeléctricos. Entre las principales actividades desarrolladas se encuentran: educación ambiental, mejoramiento del nivel de ciencias en educación, capacitación técnica comunitaria, e implementación de centros de información.

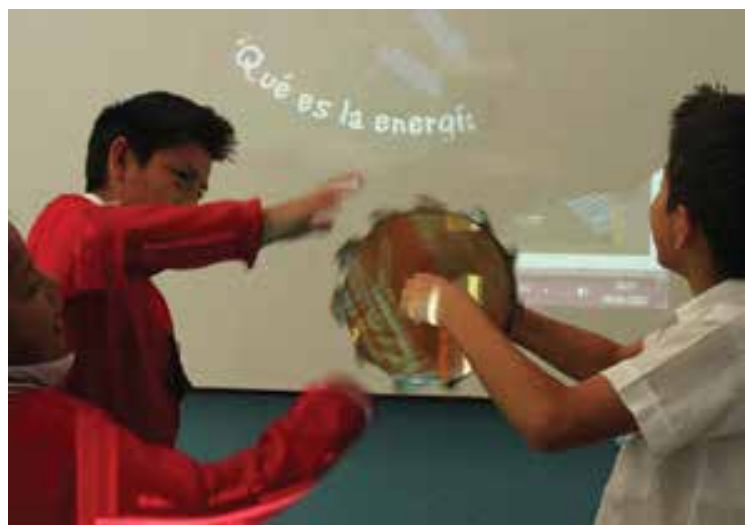

Fotografía 2: Taller "Usos de la Energía”. Escuela Albino del Curto. Cantón Limón Indanza. Fuente: PHRZS

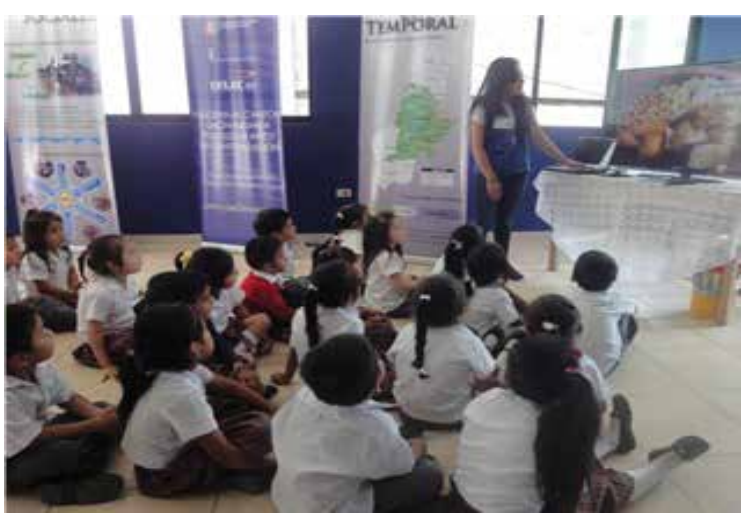

Fotografía 3: Visita de estudiantes al Centros de Información Cantón Limón Indanza. PHRZS. Fuente: PHRZS

\section{Eje 3: Manejo de la Subcuenca Hidrográfica}

Encaminado a la protección de los recursos hídricos en las áreas de influencia de los proyectos. Sus acciones fundamentales, con la participación comunitaria, son: 
- Revegetación y reforestación de las franjas de amortiguamiento de los embalses y en la cuenca hidrográfica

- Acuerdos comunitarios para el cuidado de bosques primarios y páramos

- Apoyo en el manejo de parques nacionales colindantes con las áreas de los proyectos

- Monitoreo y limpieza de embalses

- Investigación en recursos hídricos y cambio climático

\section{Eje 4: Sistema de Calidad Ambiental}

Tiene como objetivos fundamentales: colaborar en la implementación y mantenimiento de los Sistemas Integrados de Gestión; implementar los procesos para el cumplimiento de la Legislación Ecuatoriana y asesorar y monitorear los procesos de estudios y construcción de proyectos hidroeléctricos.

\section{EL ACCESO AL SERVICIO PÚBLICO DE ENERGIA A PARTIR DE UN PROYECTO DE GENERACIÓN}

Como se indicó anteriormente, CELEC EP HIDROPAUTE tiene a cargo el Proyecto Hidroeléctrico Río Zamora-Santiago, que se encuentra en etapa de estudios. El proyecto está ubicado en la provincia de Morona Santiago, que cuenta con el 63,9\% de viviendas con servicio de energía eléctrica.

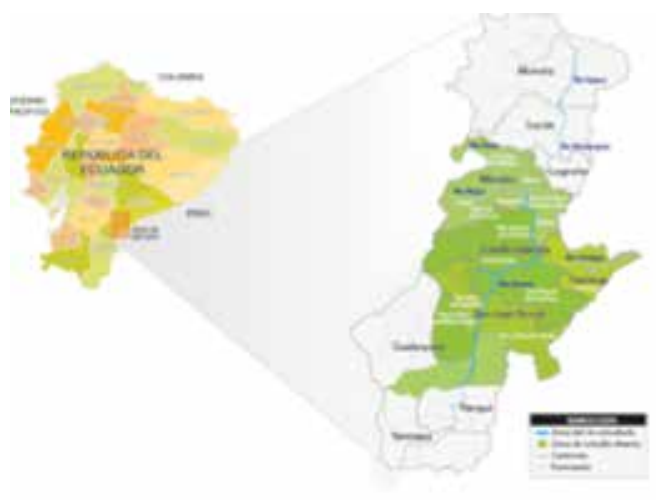

Figura 1: Mapa de ubicación del Proyecto Hidroeléctrico Río Zamora - Santiago. Fuente: PHRZS
Como parte de la metodología para el desarrollo del proyecto se han establecido mecanismos de comunicación muy cercanos a la comunidad, esto con la finalidad de informar sobre los alcances y avances del proyecto además de solventar inquietudes y temores que surgen alrededor de los mismos. En los trabajos de socialización y levantamiento de información se identificaron 94 comunidades en las cuales se aplicaron:

- 565 fichas de encuesta a la población dentro del área de influencia directa de los ríos Zamora y Namangoza.

- 680 fichas de encuesta a la población dentro del área de entorno inmediato (área de influencia social).

- 130 talleres comunitarios

- Fichas de descripción comunitarias.

- Fichas de actores sociales

- Entrevistas no estructuradas

La aplicación de las diferentes estrategias nombradas y su complemento con información secundaria permitió realizar un diagnóstico de la situación social y económica del área de estudio.

\subsection{Expectativas comunitarias frente al Proyecto Hidroeléctrico}

El proceso de levantamiento de información y socialización del proyecto permitió el contacto con líderes comunitarios, la realización de asambleas, visitas domiciliarias, reuniones con organizaciones, etc., donde se informa a la comunidad y también se reciben sus expectativas locales de desarrollo y sus inquietudes y comentarios, entre los que se destaca aquel de que "el proyecto es bueno para el país pero la electricidad y los servicios no llegan a sus comunidades".

Tal y como lo expresa el eje inclusivo del Plan Nacional de Electrificación es necesario "Armonizar el desarrollo y operación de la infraestructura del sector eléctrico con las expectativas de las comunidades". Se ha creído muy enriquecedor poder compartir algunas expresiones textuales de los habitantes del área del proyecto y un resumen de sus expectativas principales alrededor del mismo. 


\begin{tabular}{|c|c|}
\hline Comunidad & Expectativa \\
\hline Sarambitza & 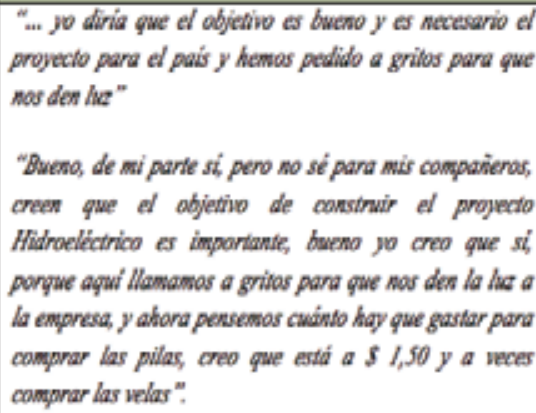 \\
\hline Ampakai & 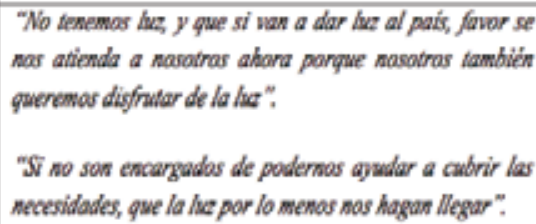 \\
\hline La Orquidea & 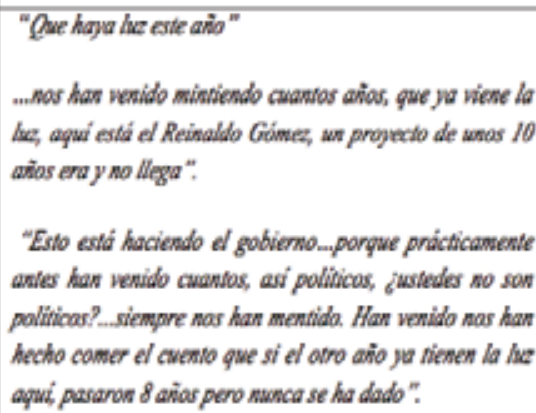 \\
\hline
\end{tabular}

Del trabajo de socialización en 70 de las 94 comunidades del área de influencia se ha obtenido una priorización de las principales necesidades expuestas que solicitan sean atendidas durante el desarrollo del proyecto. La Fig. 2 muestra en porcentajes la priorización de expectativas realizada por las comunidades.

\section{Necesidades priorizadas por las comunidades}

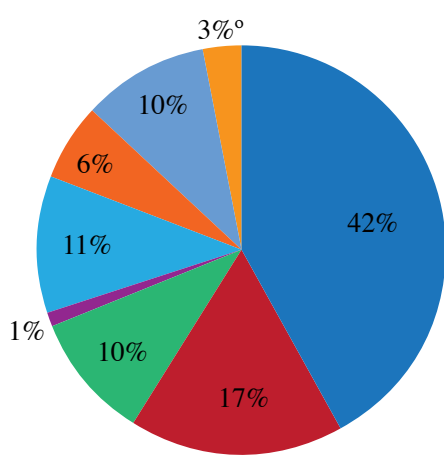

- Servicios básicos

- Atención en salud

₫ Crédito

- Infraestuctura

- Capacitación

ـEmpleo

-Comercialización

- Asesoramiento Agrícola

Figura 2: Priorización de necesidades comunitarias. Fuente: PHRZS

Se muestra que el $42 \%$ de las comunidades tiene como prioridad el desarrollo de servicios básicos, entre los cuales está el servicio de energía eléctrica.
Esta información es relevante para responder a la pregunta de por qué una empresa generadora debía involucrarse en la electrificación de las comunidades del área de influencia de sus proyectos: al igual que con otros proyectos de servicios básicos (agua potable, alcantarillado, etc.) el acceso al servicio de electrificación rural es un derecho reclamado durante años por los habitantes de la zona quienes ven a los desarrolladores de proyectos eléctricos como los llamados a satisfacer esa demanda, sin importar si tienen la competencia legal para hacerlo. El no involucrarse en este aspecto puede poner en riesgo las relaciones con la comunidad y, por ende, el éxito mismo del proyecto de generación.

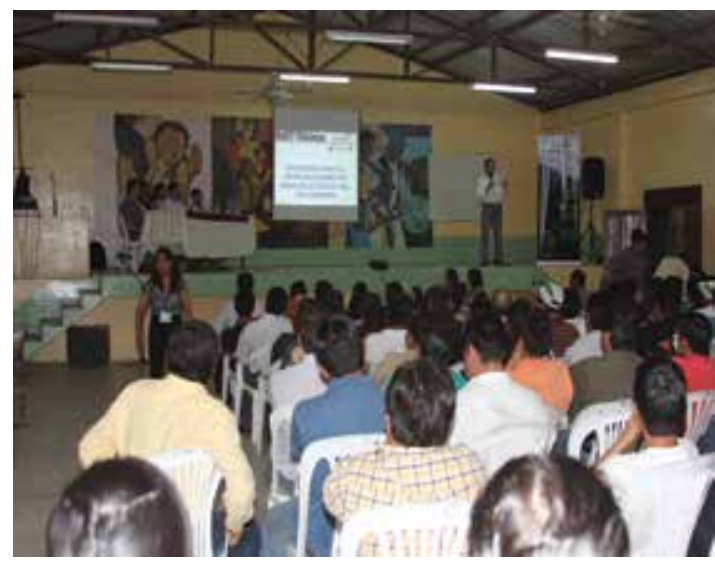

Fotografía 4: Taller de socialización del Proyecto

Hidroeléctrico Río Zamora-Santiago. Cantón San Juan Bosco. Fuente: PHRZS

\subsection{La Electrificación Rural desde la Gestión Socio-Ambiental.}

Una de las estrategias fundamentales de la inversión pública está encaminada a satisfacer la dotación de bienes y servicios esenciales, como es el caso de la electricidad, dotación que permite mejorar y salvaguardar las capacidades de la sociedad en su conjunto y de los individuos que la conforman.

Los proyectos de generación eléctrica, con sus respectivos planes de manejo y medidas compensatorias, ejecutados de forma aislada, si bien pueden cumplir con la normativa vigente no garantizan un adecuado desarrollo de los territorios donde se asientan dichos proyectos. Lo paradójico es que las comunidades exigen a los promotores de proyectos mejoras en su nivel de vida. Ello demanda acciones públicas, con un enfoque intersectorial y de derechos, que trasciende la responsabilidad de "construir un proyecto" y lleva a los promotores a involucrarse en procesos de desarrollo a través de la prestación de servicios integrales e integrados. Las intervenciones coordinadas y respetando el ámbito de competencias, pueden ser realizadas en las diferentes etapas de estudio, construcción y operación de los proyectos. 
Dentro del Eje 1 de Gestión Ambiental de CELEC EP HIDROPAUTE “Apoyo al Desarrollo Territorial" se considera la implementación de proyectos de desarrollo comunitario como aquellos relacionados a la electrificación. Para el caso del Proyecto Hidroeléctrico Río Zamora-Santiago se ha contemplado la ejecución de proyectos de electrificación rural dentro de su área de influencia, a través de la firma de convenios de cooperación entre CELEC EP y la empresa distribuidora de energía, Empresa Eléctrica Regional CENTROSUR. En una primera etapa se contemplaron 8 proyectos de alumbrado público y 12 proyectos de electrificación rural, brindando servicio a 17 comunidades. Se debe indicar que para la selección de los proyectos a ser ejecutados se involucró a los representantes de los Gobiernos Autónomos Descentralizados y aun cuando los proyectos superaron los límites de financiamiento ${ }^{1}$, se los consideró viables debido a la necesidad de cubrir un déficit histórico en las comunidades que deben contar con el servicio de energía eléctrica. El siguiente gráfico muestra, en síntesis, los pasos para la construcción de los proyectos de electrificación rural:

Levantamiento de línea base (situación

actual) de comunidades de área de

influencia. Expectativas comunitarias.
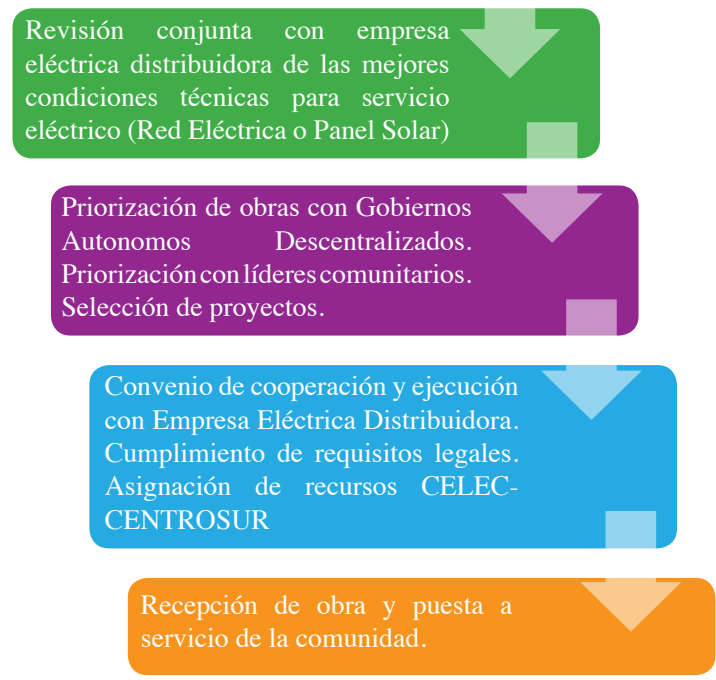

Los 20 proyectos ejecutados hasta julio de 2013 incorporan 205 nuevos clientes, 820 beneficiarios en total. Se tiene previsto además una segunda etapa donde se estiman 120 clientes y 500 beneficiarios.

1 De conformidad con los criterios vigentes para la selección de proyectos FERUM

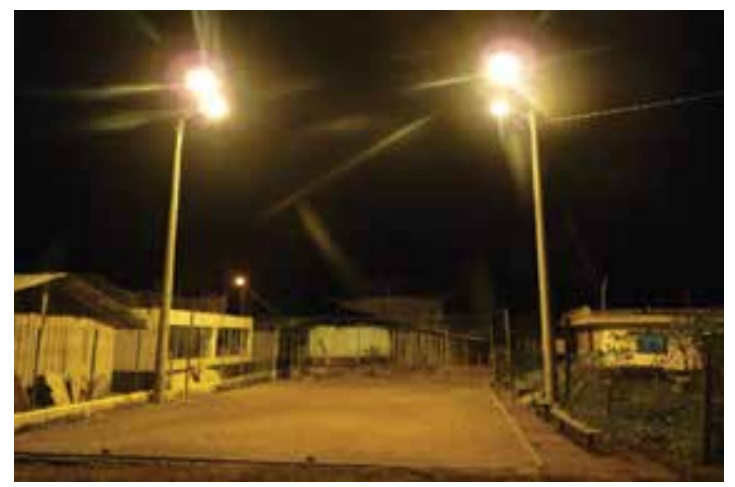

Fotografía 5: Iluminación de cancha deportiva de Santiago de Panantza. Cantón San Juan Bosco. Fuente: PHRZS

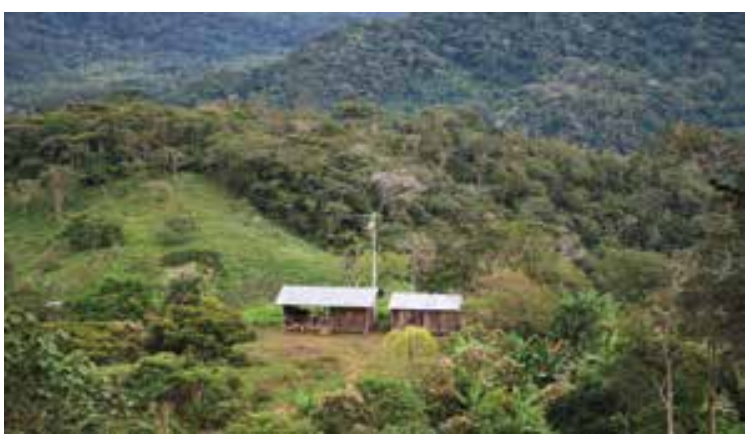

Fotografía 6 :Usuario de proyecto de electrificación. Parroquia Bomboa. Cantón San Juan Bosco. Fuente: PHRZS

La Tabla 1 muestra las comunidades atendidas con los proyectos de electrificación en la provincia de Morona Santiago:

Tabla 1:Lista de Proyectos de electrificación PHRZS

\begin{tabular}{|c|c|c|}
\hline Nombre del Progwerta Camunidad & Casandn & 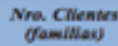 \\
\hline Santa Rosa, Asaa, Yaa, Nayup & San Juan Bosco & 59 \\
\hline Kunakus & San Jaun Boseo & $2 z$ \\
\hline Nambar & San Jwan Alosce & 6 \\
\hline Afwlticenscha cerrada y villey descublenta & San Juan Bosco & Comanitario \\
\hline Afultricancha cerrada y willoy descublerna & San Juan Josce & Comunitario \\
\hline Mwltinawe ha cerrada 27 de noviembion & San Juan aloseo & Comanihario \\
\hline Mwhinicancha cerroda Santa Rosa & San Juan Basco & Comanikario \\
\hline Mwhlicancha cerrada La Libertad & San Juan Bosco & Comanikario \\
\hline Mwlicancha cerrada San Muen Boseo & San Juan Bosce & Comwnikario \\
\hline Mntrisencha cerrada y abverta Wacembers & Saw Juan Baseo & Comanikario \\
\hline Maminacioin entrada narie de Limoin & Limole & Comaniario \\
\hline Naranywso & Limón & $3 t$ \\
\hline Goucamayos & Limole & 29 \\
\hline Valle de Chimandaz & Limde & II \\
\hline Coangas & Limón & 10 \\
\hline nuads & Limoln & $2 s$ \\
\hline Partideno & Santioges & Is \\
\hline Puenne Guajugquil - via parnidero & Sannilegeo & 9 \\
\hline Puente Gomapaquil & Santlageso & s \\
\hline Singuiantza, Yweal, Tiendeaskas & Santlages & g \\
\hline
\end{tabular}




\section{CONCLUSIONES Y RECOMENDACIONES}

Hoy en día en el Ecuador, los proyectos de generación eléctrica, en cualquiera de sus etapas (estudios, construcción u operación) no pueden ser ejecutados de forma aislada, cumpliendo únicamente la normativa vigente, sin considerar la interacción con los territorios donde se asientan dichos proyectos. Las comunidades involucradas demandan mejoras en su nivel de vida lo cual lleva a los promotores de los proyectos a involucrarse en los procesos de desarrollo territorial por medio de intervenciones directas o coordinadas, respetando el ámbito de competencias institucionales.

Es importante para una empresa de generación, contar con una estructura definida y un equipo técnico suficiente que haga frente a las demandas comunitarias, respondiendo a una política de responsabilidad socioambiental claramente establecida. En el caso de CELEC EP - HIDROPAUTE y su proyecto Río Zamora -Santiago, queda claro que la responsabilidad socioambiental de la Empresa se vio abocada a responder a una demanda específica: el acceso de la comunidad al servicio de electrificación. La atención oportuna de esta demanda, que es a su vez un derecho de la población, no solo que mejora las relaciones con la comunidad sino además permite desarrollar el proyecto principal en un ambiente de mayor confianza, incrementando su posibilidad de éxito del proyecto.

Así, a la pregunta de por qué una empresa generadora debería involucrarse en la electrificación de las comunidades del área de influencia de sus proyectos, a pesar de no ser su competencia, se podría responder con lo siguiente: al igual que con otros servicios básicos (agua potable o alcantarillado) el acceso al servicio de electrificación a más de una necesidad es un derecho de las personas, y son las comunidades quienes tienen el poder de legitimar (o no) al proyecto en desarrollo, en la medida que dicho proyecto aporte a satisfacer esas necesidades.

\section{REFERENCIAS BIBLIOGRÁFICAS}

[1] Constitución de la República del Ecuador.

[2] Plan Nacional del Buen Vivir 2009-2013.

[3] CONELEC (2010) Plan Maestro de Electrificación 2009-2020.

[4] Albornoz, E., (2012) Presentación MEER. Visión del Sector Eléctrico Ecuatoriano.

[5] Guzmán, P. y Espinoza, J.L. (2010), Gestión Socio-Ambiental en el Proyecto Mazar, Revista Interconexiones, Diciembre, No. 75 Año XX: 5053.

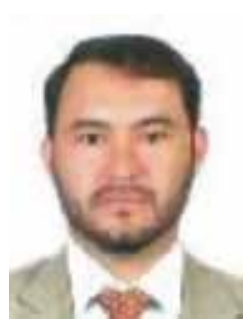

Luis Alberto Ochoa Pesantez (1976) es ingeniero eléctrico graduado en la Universidad de Cuenca y posee una Maestría en Administración de Empresas de la Universidad del Azuay Ecuador y un Diplomado en Gestión de Proyectos Gubernamentales de la UNAM

México.

Ha trabajado como profesor en la Facultad de Ingeniería Eléctrica en la Universidad Politécnica Salesiana, ingeniero de la Dirección de Planificación de la Empresa Eléctrica CENTROSUR, coordinador del Proyecto Hidroeléctrico Minas- Jubones (300 MW), Director de Gestión Técnica Corporación Eléctrica del Ecuador CELEC S.A, 2010-2012, subgerente de Planificación y Procesos de la Unidad de Negocio HIDROPAUTE - CELEC EP y actualmente Director Proyecto Hidroeléctrico Río Zamora-Santiago.

Ha realizado investigación para la optimización en diferentes aspectos de los Sistemas Eléctricos de Potencia. Expositor investigador en "Seminarios de Distribución y Comercialización de Energía Eléctrica", organizados por la ECUACIER 


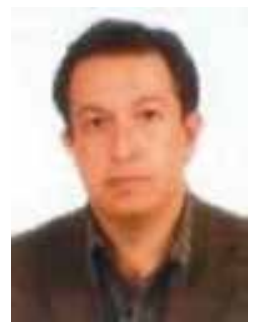

Paul Esteban Martinez Mosquera (1972) es ingeniero eléctrico graduado en la Universidad de Cuenca y posee una Maestría en Gestión Tecnológica yun Diplomado en Auditorías Ambientales.

Ha trabajado como Gerente del Proyecto Unidades Educativas del Milenio del Ministerio de Educación, Director Ejecutivo de la Unidad Educativa CEDFI, Gerente de Electrónica Control y Sistemas Cía. Ltda.

Además, ha trabajado como consultor técnico en el sector privado. Actualmente trabaja como Especialista Ambiental de la Unidad de Gestión Ambiental de HIDROPAUTE, Proyecto Hidroeléctrico Río ZamoraSantiago.

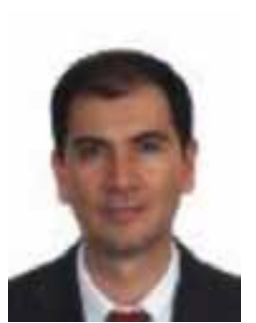

Juan Leonardo Espinoza, (1967) es ingeniero eléctrico graduado en la Universidad de Cuenca, tiene una Maestría en Energía y Ambiente del Programa de la Universidad de Calgary y la OLADE. Obtuvo su Ph. D. en la Universidad de Calgary, Canadá, con doble especialidad en Desarrollo Sustentable/Gestión Ambiental y Estrategia. Del 2005 al 2009, trabajó en el Municipio de Cuenca como Director de la Comisión de Gestión Ambiental. En el 2009 fue Subsecretario de Energía Renovable y desde el 2010 laboró en CELEC EP - HIDROPAUTE, donde ocupó el cargo de Gerente de febrero 2011 a junio de 2013.

Ha dictado cursos, principalmente de postgrado, sobre Energía, Gestión Ambiental y Estrategia en la OLADE, Universidad de Calgary y en diferentes universidades nacionales. Sus publicaciones y trabajos de investigación han sido presentados en eventos académicos en varios países. Actualmente, es profesor principal en la Facultad de Ingeniería de la Universidad de Cuenca. 


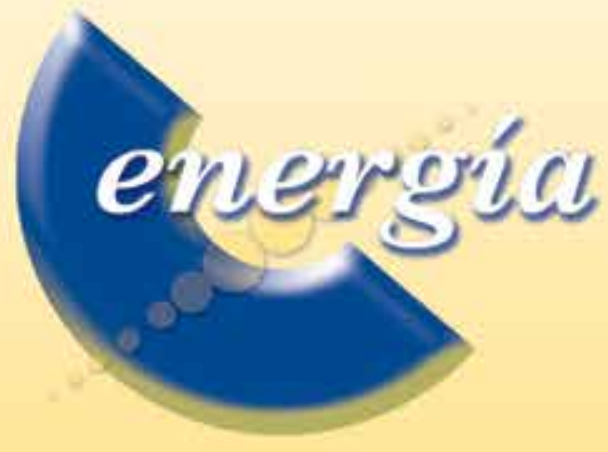

\section{Artículos Premiados Ediciones Anteriores}

Revista Técnica "energía" se despliega en un entorno global, patrocina la investigación y fomenta el intercambio de ideas encaminadas al beneficio y desarrollo del Sector Eléctrico, trata sobre avances científicos y tecnológicos, así como, experiencias de alto nivel en Sistemas Eléctricos de Potencia, Mercados Eléctricos y en las Interconexiones Internacionales de Electricidad.

La revista cumple una década y marca un hito importante en la publicación sobre el desarrollo técnico, no solo del Sector Eléctrico, sino del país.

En las 10 ediciones de la revista se han recopilado 182 trabajos técnicos sobre investigaciones y desarrollos tecnológicos de interés institucional y trascendencia a nivel del Sector Eléctrico. En las tres primeras ediciones, los autores participantes fueron únicamente funcionarios de CENACE; a partir de la cuarta edición se invitó a personas relacionadas con el Sector Eléctrico; y a partir de el año 2008 se premiaron a los mejores trabajos de cada edición.

El presente anexo contiene una recopilación de aquellos trabajos galardonados en las ediciones anteriores, y constituye una conmemoración por el décimo aniversario de la revista. Estos trabajos no forman parte de la edición $\mathrm{N}^{\circ} 10$. 